\title{
CONSUMO DE GAS NATURAL EN BOLIVIA: UNA APLICACION DEL SISTEMA CUADRATICO CASI IDEAL DE DEMANDA
}

NATURAL GAS CONSUMPTION IN BOLIVIA: A QUADRATIC ALMOST IDEAL DEMAND SYSTEM APPLICATION

\section{MAURICIO MEDINACELI MONRROY*}

Organización Latinoamericana de Energía (OLADE)

\begin{abstract}
This document tries to quantify the possible impacts, on the natural gas consumption, of changes in the flat sale price, to the final consumer, for the natural gas distribution system in Bolivia, in particular, it was analyzed the progressivity of this policy on households from eight urban cities. The results obtained through the 'Quadratic Almost Ideal Demand System' (QUAIDS) suggest that, a lineal decrease of the natural gas price for all the families presents an important regressive component. The reason is clear, high income families can, with high probability, modify its durable goods portfolio and take advantage of the benefits from reduced prices. In this sense, non lineal prices could be an attractive instrument to achieve bigger progressivity inside the tariffs system on the natural gas distribution system in Bolivia.
\end{abstract}

Keywords: QUAIDS, natural gas, energy price.

JEL Classification: $D 1 ; D 3 ; D 6 ; Q 4$.

* Coordinador de hidrocarburos de la Organización Latinoamericana de Energía (OLADE). Master en Economía y ex Ministro de Hidrocarburos de Bolivia. Se ha desempeñado en funciones directivas y de asesoría tanto en empresas públicas como privadas y entidades internacionales tales como Yacimientos Petrolíferos Fiscales Bolivianos (YPFB), la Cámara Boliviana de Hidrocarburos, la CAF, el Banco Mundial y el Banco Interamericano de Desarrollo (BID). Docente Universitario y autor de varios libros, artículos y boletines especializados en el tema energético. Email: mmedinaceli@yahoo.com 


\section{Resumen}

Este documento intenta cuantificar los posibles impactos, sobre el consumo de gas natural, de cambios en el precio de venta al consumidor final dentro el sistema de distribución de gas natural en Bolivia; en particular, se analizará la progresividad de esta medida sobre los hogares de ocho ciudades del sector urbano. Los resultados obtenidos a través del 'Sistema Cuadrático Casi Ideal de Demanda' (QUAIDS) sugieren que disminuir el precio del gas natural de manera lineal para todas las familias, como medida para incentivar su consumo, contiene un componente regresivo importante. La razón es clara, son las familias de elevado ingreso las que, con alta probabilidad, pueden reconvertir su portafolio de bienes duraderos y así aprovechar los beneficios de menores precios en el gas natural. En este sentido, esquemas no lineales de precios podrían constituirse en un instrumento atractivo para lograr mayor progresividad en el sistema de tarifación de estas redes.

Palabras Clave: QUAIDS, gas natural, precios energía.

Clasificación JEL: D1; D3; D6; $Q 4$.

\section{INTRODUCCION}

No son pocas las veces que la política energética en Bolivia hace énfasis en el gasto que realiza el Estado en la ampliación de redes de gas natural. ${ }^{1}$ De hecho, existe la percepción casi generalizada de que dicha ampliación, sobre todo en el sector residencial (familiar), tendrá un efecto positivo sobre el bienestar de las personas, toda vez que el gasto en este energético es menor a las alternativas, en particular, la energía eléctrica y en segundo lugar del Gas Licuado de Petróleo (GLP). Así como también no es nuevo el compromiso de las autoridades en Bolivia por disminuir el precio del gas natural en todo el país. ${ }^{2}$ Entonces, bien vale la pena preguntarse si este tipo de políticas beneficia a los sectores de menor ingreso de la población, dado que la cantidad de dinero que generalmente se destina a este tipo de proyectos no es trivial.

Con el objetivo central de conocer cuáles son las principales características del consumo de gas natural domiciliario, en este trabajo se estima la función de demanda por este producto. En particular, utilizando la información proveniente de las encuestas de hogares realizadas por el Instituto Nacional de Estadística de Bolivia, se estima el

\footnotetext{
1 "Yacimientos Petrolíferos Fiscales Bolivianos (YPFB) reactivará conexiones de gas natural a domicilio con una inversión de 100 millones de dólares". Periódico Boliviano "El Diario", 29 de abril de 2009.

2 "Si antes Emcogas [empresa distribuidora de gas natural] cobraba 18,28 bolivianos, ahora que hemos recuperado y está en Yacimientos, la tarifa, el costo será de 8 bolivianos para todo el país”, Evo Morales - Periódico Boliviano “La Razón”, 22 de julio de 2009.
} 
sistema cuadrático casi ideal de demanda (QUAIDS por sus siglas en inglés) propuesto originalmente por Deaton \& Muellbauer (1980).

En varias oportunidades se menciona la necesidad de masificar el consumo de gas natural a través de menores precios de éste y/o incrementos en el precio de su sustituto más cercano, el gas licuado de petróleo (GLP). Por este motivo, el presente trabajo analizará el cambio en el bienestar de las familias con distintos niveles de precios para el gas natural distribuido por redes.

El principal resultado encontrado es que el consumo de gas natural actualmente está concentrado en familias de ingreso elevado, hecho que puede deberse a la capacidad de reconvertir su portafolio de bienes duraderos y así utilizar el gas natural intensivamente. Por ello, una disminución uniforme en el precio de venta del gas natural destinado al consumo familiar, tendrá un componente regresivo no despreciable. En este sentido, revisar nuevamente la priorización del gasto público es una tarea central.

El texto está ordenado como sigue: en la primera sección se describe el contexto general del sector hidrocarburos en Bolivia; en la segunda se presentan algunas cifras someras sobre el consumo del gas natural en el mercado interno; en la tercera se describen las principales características de los datos utilizados en el modelo; luego se presentan el modelo y los principales resultados; finalmente se detallan las principales conclusiones del trabajo. Como es usual, cualquier error u omisión es responsabilidad completa del autor.

\section{REDES DE DISTRIBUCION DE GAS NATURAL}

Como parte del proceso de apertura, dentro del sector hidrocarburos, que inició Bolivia al comenzar la década de los noventa, la empresa estatal de hidrocarburos en Bolivia, Yacimientos Petrolíferos Fiscales Bolivianos (YPFB), decidió abrir el espacio necesario para que el sector privado invierta en las redes de distribución de gas natural. De esta forma, se otorgaron concesiones por veinte años a sociedades empresariales que deberían, a partir de la firma del contrato, operar, mantener y ampliar las redes de distribución de gas natural en los principales centros urbanos del país. Es así que se crean las siguientes empresas:

- EMCOGAS encargada de la distribución en Cochabamba, Quillacollo y Cliza.

- EMDIGAS abastece a Sucre.

- EMTAGAS en una empresa mixta (con participación de los Gobiernos Locales) que abastece a las ciudades de Bermejo, Tarija, Villamontes y Yacuiba.

- $\quad$ SERGAS abastece a Santa Cruz de la Sierra, Warnes, Montero y Minero.

Por otra parte, YPFB quedó a cargo del abastecimiento en las ciudades de La Paz, El Alto, Viacha, Oruro, Potosí y Camiri, ya que el proceso de transferencia al sector privado no pudo completarse en el plazo correspondiente. De esta manera, el occidente del país tiene a la empresa estatal como distribuidora de gas natural, quedando en el oriente las empresas privadas mencionadas anteriormente, sin embargo, la concesión de varias de ellas está por concluir. 
La Tabla 1 presenta el consumo de gas natural por redes para el período 1998-2007, desagregada por empresa distribuidora. De ella se desprende que son las empresas de Cochabamba y Santa Cruz aquellas que presentan mayores tasas de crecimiento anuales, $12 \%$ y $11 \%$ respectivamente, le siguen el Eje Occidental (10\%), Tarija (5,5\%) y Sucre (4,8\%); finalmente, se observa que la tasa de crecimiento promedio anual a nivel nacional es $10,4 \%$, cifra mayor a la tasa de crecimiento del PIB o de la población.

\section{TABLA 1}

CONSUMO DE GAS NATURAL POR EMPRESA DISTRIBUIDORA (MM PC)

\begin{tabular}{|l|c|c|c|c|c|c|c|c|c|}
\hline $\begin{array}{c}\text { Empresa } \\
\text { distribuidora }\end{array}$ & 2000 & 2001 & 2002 & 2003 & 2004 & 2005 & 2006 & 2007 & $\begin{array}{c}\text { Crecimiento } \\
\text { anual }\end{array}$ \\
\hline EMCOGAS & 4.139 & 4.224 & 5.193 & 5.407 & 6.261 & 7.372 & 8.107 & 9.209 & $12,1 \%$ \\
SERGAS & 4.026 & 4.007 & 3.967 & 4.714 & 5.181 & 6.151 & 6.749 & 8.516 & $11,3 \%$ \\
$\begin{array}{l}\text { EMDIGAS y } \\
\quad \text { COSERMO }\end{array}$ & 1.258 & 728 & 1.027 & 1.224 & 1.345 & 1.421 & 1.681 & 1.757 & $4,9 \%$ \\
EMTAGAS & 1.030 & 947 & 938 & 944 & 1.029 & 1.249 & 1.333 & 1.501 & $5,5 \%$ \\
YPFB & 4.427 & 4.810 & 5.370 & 5.512 & 6.095 & 6.202 & 7.638 & 8.692 & $10,1 \%$ \\
\hline Total nacional & 14.880 & 14.716 & 16.495 & 17.800 & 19.911 & 22.395 & 25.508 & 29.676 & $10,4 \%$ \\
\hline
\end{tabular}

Fuente: Superintendencia de Hidrocarburos.

Elaboración: Propia.

La Tabla 2 presenta el total nacional desagregado por categoría de consumo. Como es natural en mercados emergentes, como el boliviano, el consumo está concentrado en el sector industrial; sin embargo, el sector vehicular a través del consumo de gas natural vehicular (GNV) presenta el mayor crecimiento de todas las categorías, luego le siguen el sector residencial, comercial e industrial.

TABLA 2

CONSUMO DE GAS NATURAL POR CATEGORIA (MM PC)

\begin{tabular}{|l|r|r|r|r|r|r|r|r|r|}
\hline $\begin{array}{c}\text { Empresa } \\
\text { distribuidora }\end{array}$ & 2000 & 2001 & 2002 & 2003 & 2004 & 2005 & 2006 & 2007 & $\begin{array}{c}\text { Crecimiento } \\
\text { anual }\end{array}$ \\
\hline Industrial & 13.517 & 12.705 & 13.804 & 14.275 & 15.215 & 16.038 & 17.312 & 18.971 & $5,0 \%$ \\
GNV & 931 & 1.465 & 2.035 & 2.667 & 3.582 & 5.040 & 6.699 & 8.999 & $38,3 \%$ \\
Comercial & 262 & 324 & 690 & 455 & 532 & 617 & 698 & 779 & $16,9 \%$ \\
Doméstico & 170 & 222 & 267 & 403 & 581 & 700 & 798 & 927 & $27,4 \%$ \\
\hline Total nacional & 14.880 & 14.716 & 16.795 & 17.800 & 19.911 & 22.395 & 25.508 & 29.676 & $10,4 \%$ \\
\hline
\end{tabular}

Fuente: Superintendencia de Hidrocarburos.

Elaboración: Propia. 
Durante los años 2009 y 2010 finalizarán las concesiones otorgadas al sector privado para la administración de las redes de gas natural situadas en la parte oriental del país. Dado el proceso nacionalizador que en este momento tiene la República de Bolivia, a medida que las concesiones finalicen, YPFB se espera que sea la empresa encargada de operar las redes de distribución de gas natural en todo el país. Por este motivo, las políticas de expansión de dichas redes estarán a cargo del Estado a través de YPFB, sin embargo, en algunas regiones, por ejemplo Tarija, la participación accionaria del Gobierno Departamental es importante.

\section{DESCRIPCION DE DATOS}

En esta sección se describen los datos utilizados en el modelo, en particular, se presentan las principales estadísticas de las variables insertas en las encuestas de hogares y se describen los precios seleccionados.

\section{Encuestas de Hogares}

La base de datos se obtuvo de las Encuestas a Hogares dentro del Programa de Mejoramiento de Encuestas y Medición de Condiciones de Vida (MECOVI), que periódicamente lleva a cabo el Instituto Nacional de Estadística de la República de Bolivia (INE). En esta encuesta se realizan preguntas que cubren las principales características del hogar, respecto a empleo, salud, educación, infraestructura, etc. En este documento se utilizaron los resultados a las siguientes preguntas:

- ¿Qué tipo de combustible o energía utiliza para cocinar?

- ¿Cuánto gasta normalmente en el combustible que utiliza para cocinar al mes?

El período de análisis es 1999-2007, con excepción de 2003 y 2004; en el resto de años se realizó la encuesta durante el mes de noviembre de cada uno de ellos. En el caso del período 2003-2004, el INE hizo esta encuesta a lo largo del año, por ello, se tiene una sola base de datos en ambos períodos.

Puesto que la red de distribución de gas natural se encuentra concentrada en las principales capitales de los departamentos en Bolivia, el estudio se concentró en la información proveniente del área urbana en: La Paz, Cochabamba, Santa Cruz de la Sierra, Oruro, Potosí, Tarija y Sucre. La Tabla 3 presenta el número de encuestas por familia utilizadas en este estudio y el porcentaje que ellas representan del universo total de familias.

Por otra parte, es importante señalar que el Instituto Nacional de Estadística proporciona un factor de expansión que permite reproducir el universo total de familias observado en el censo de población y vivienda del año 2001, ajustado por una determinada tasa de crecimiento. Debido a restricciones de hardware, el factor de expansión utilizado en este estudio fue ajustado en la escala 1:100, de su valor original. Dado que se mantuvo la proporcionalidad original, este cambio de escala 
no debería afectar los resultados econométricos realizados posteriormente, ver Lee y Forthofer (2006).

TABLA 3

NUMERO DE ENCUESTAS Y REPRESENTATIVIDAD

\begin{tabular}{|l|c|c|}
\hline \multicolumn{1}{|c|}{ Gestión } & Número de encuestas & Porcentaje del universo \\
\hline 1999 & 1.467 & $0,14 \%$ \\
2000 & 2.498 & $0,21 \%$ \\
2001 & 2.694 & $0,23 \%$ \\
2002 & 3.018 & $0,25 \%$ \\
$2003-2004$ & 6.519 & $0,47 \%$ \\
2005 & 2.122 & $0,15 \%$ \\
2006 & 2.478 & $0,17 \%$ \\
2007 & 2.307 & $0,17 \%$ \\
\hline
\end{tabular}

Fuente: Encuestas de Hogares del Programa MECOVI.

Elaboración: Propia.

Debido a la especificación del modelo econométrico, estudiar las principales características de los datos utilizados se facilita mucho si la muestra se divide en dos: 1) familias que utilizan gas natural para cocinar y; 2) familias que utilizan gas licuado de petróleo (GLP) para cocinar. ${ }^{3}$ En este sentido, la Tabla 4 presenta las principales características del primer grupo de familias. Los datos están agrupados de acuerdo al año en que se realizó la encuesta de hogares, la primera fila presenta el promedio de la muestra ${ }^{4}$ y la segunda la desviación estándar. Por otra parte, la Tabla 5 presenta dichas características para las familias que afirman utilizar GLP en sus cocinas.

El contraste de las dos tablas anteriores proporciona aproximaciones preliminares interesantes de comentar: 1) el consumo promedio de gas natural por cada familia es mayor al consumo promedio de GLP,5 2) el gasto total de las familias que utilizan gas natural es mayor al de las familias que utilizan GLP, de ello se infiere que en promedio el primer grupo de familiar posee un ingreso familiar mayor al segundo grupo; 3 ) el porcentaje de familias que tiene vivienda propia es mayor en el grupo del gas natural $\mathrm{y} ; 4)$ el stock de activos de aquellas familias que consumen gas natural es mayor al del otro grupo. Estas cifras sugieren que las familias que utilizan gas natural consumen más energía, poseen un ingreso familiar mayor y poseen una mayor cantidad de activos durables al interior del hogar.

3 El gas natural se refiere al componente gaseoso $(\mathrm{C} 1+\mathrm{C} 2)$ de la cadena de hidrocarburos, este producto llega a los hogares únicamente a través de las redes de distribución. Por otra parte, el gas licuado de petróleo se refiere al propano y butano $(\mathrm{C} 3+\mathrm{C} 4)$ consumido por las familias en garrafas (cilindros o bombonas) de 10 kilogramos que se comercializan a través de camiones distribuidores de muy pequeña escala.

4 Siempre ajustado por el factor de expansión.

5 Ambos medidos en unidades de energía, millones de BTU. 


\begin{tabular}{|c|c|c|c|c|c|c|c|}
\hline \multirow{9}{*}{ 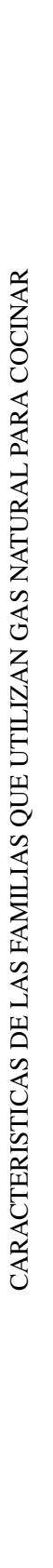 } & 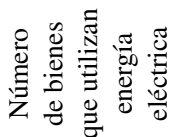 & \multicolumn{5}{|c|}{ 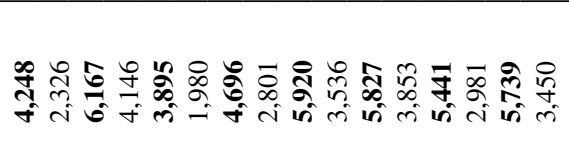 } & 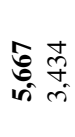 \\
\hline & 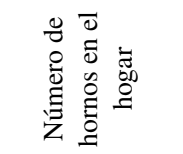 & \multicolumn{5}{|c|}{ 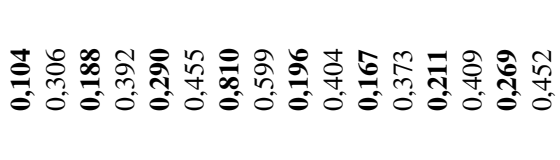 } & 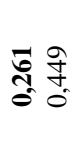 \\
\hline & 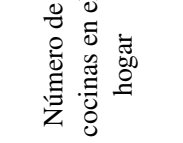 & \multicolumn{5}{|c|}{ 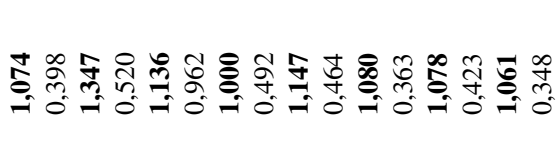 } & 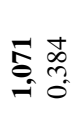 \\
\hline & 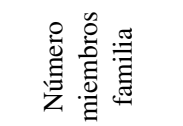 & \multicolumn{5}{|c|}{ 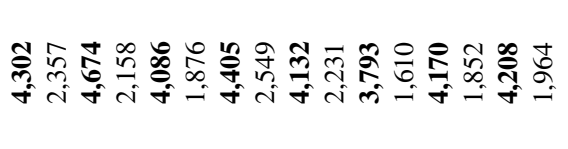 } & $\begin{array}{l}\stackrel{+}{5} \\
\Rightarrow\end{array}$ \\
\hline & 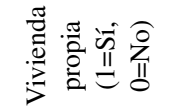 & \multicolumn{5}{|c|}{ 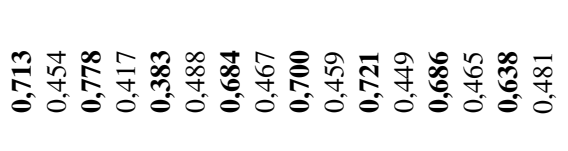 } & 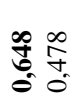 \\
\hline & 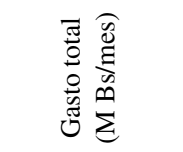 & \multicolumn{5}{|c|}{ 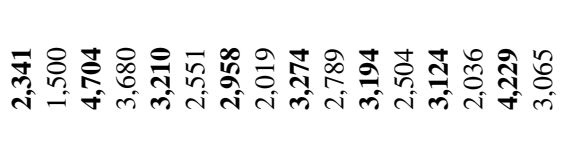 } & $\begin{array}{l}\hat{\sigma} \\
\stackrel{\hat{\sigma}}{\hat{i}} \\
\text { i }\end{array}$ \\
\hline & 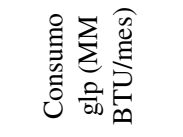 & \multicolumn{5}{|c|}{ 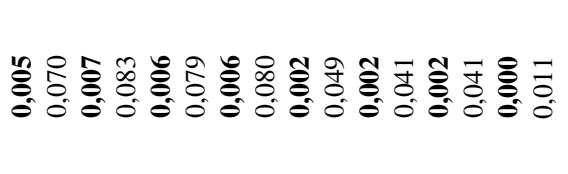 } & $\begin{array}{l}\bar{\Xi} \\
\bar{\Xi} \\
\stackrel{-}{0}\end{array}$ \\
\hline & 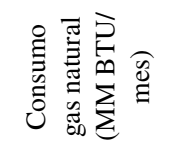 & \multicolumn{5}{|c|}{ 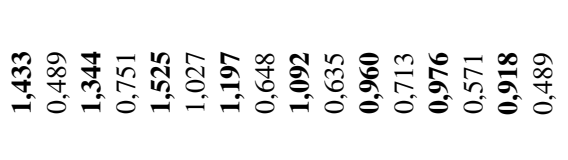 } & 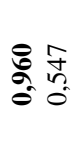 \\
\hline & 穵 & \multicolumn{6}{|c|}{ 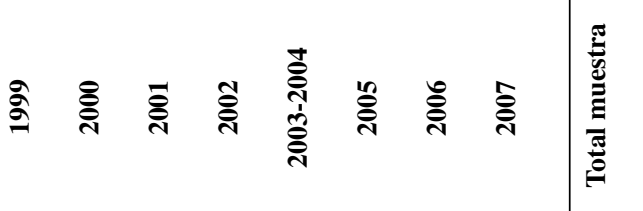 } \\
\hline
\end{tabular}




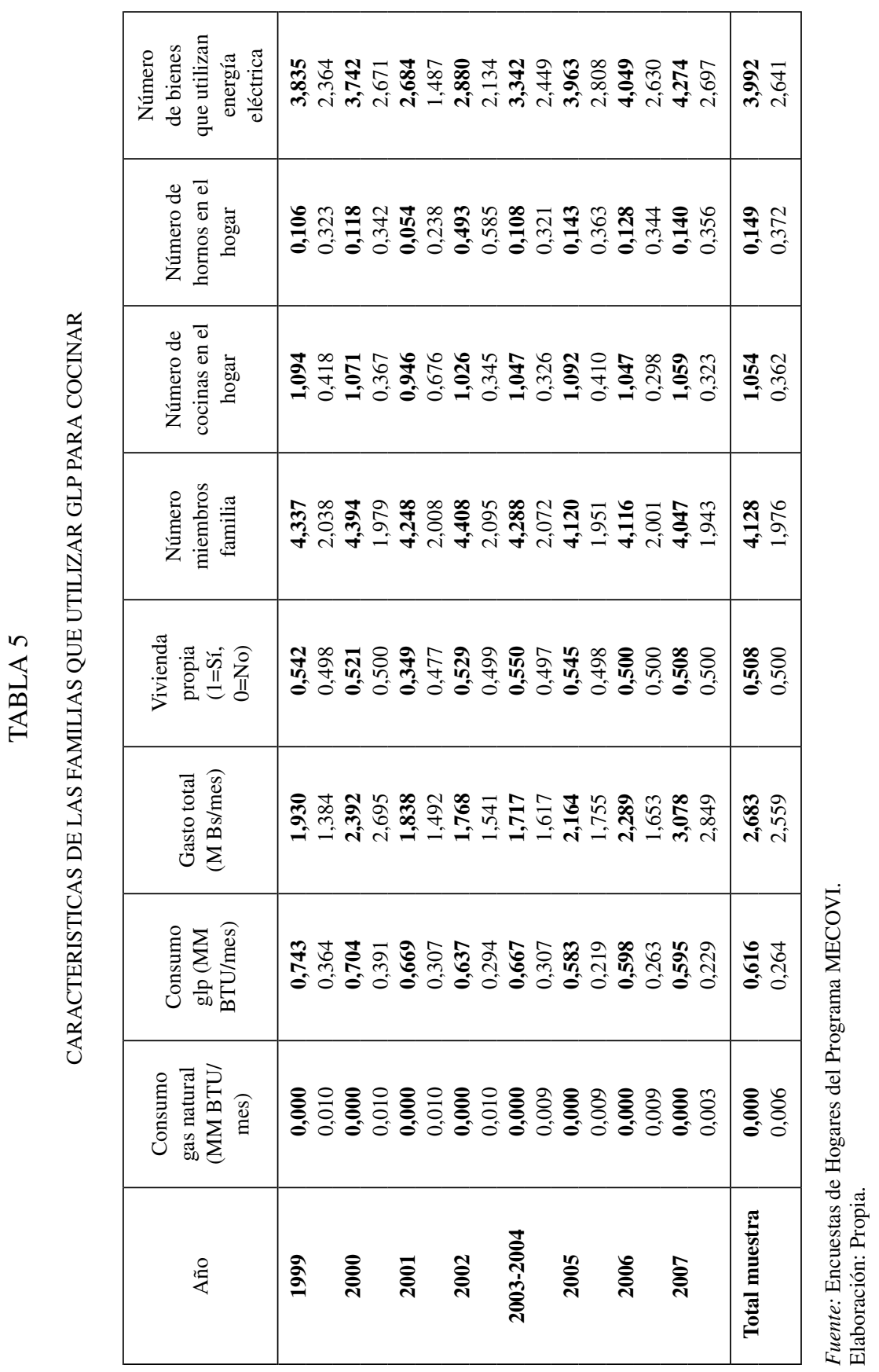


La Tabla 6 presenta el comportamiento del porcentaje de familias que afirman utilizar gas natural para cocinar, ordenados por quintil de ingreso, para toda Bolivia. Queda claro que durante el período de análisis, el incremento de la demanda residencial por gas natural se concentra en familias de elevado ingreso. Este resultado es compatible con aquellos obtenidos en Medinaceli (2005); de acuerdo a este trabajo, las familias que se benefician en mayor proporción de las instalaciones de gas natural por redes son aquellas de elevado ingreso. La razón es clara, son estas familias quienes podrán, con mayor probabilidad, reconvertir su portafolio de bienes durables para utilizar gas natural.

\section{TABLA 6}

CONSUMO DE GAS NATURAL POR QUINTIL DE INGRESO - TOTAL BOLIVIA

\begin{tabular}{|c|c|c|c|c|c|c|c|c|}
\hline Quintil & 1999 & 2000 & 2001 & 2002 & $2003-2004$ & 2005 & 2006 & 2007 \\
\hline 1 & $0,0 \%$ & $0,2 \%$ & $0,1 \%$ & $0,2 \%$ & $0,2 \%$ & $0,6 \%$ & $0,5 \%$ & $0,9 \%$ \\
2 & $0,7 \%$ & $0,3 \%$ & $0,6 \%$ & $0,5 \%$ & $0,7 \%$ & $1,1 \%$ & $0,9 \%$ & $2,9 \%$ \\
3 & $1,0 \%$ & $1,1 \%$ & $0,7 \%$ & $0,2 \%$ & $1,1 \%$ & $1,8 \%$ & $2,4 \%$ & $2,9 \%$ \\
4 & $1,1 \%$ & $0,8 \%$ & $0,8 \%$ & $0,7 \%$ & $1,1 \%$ & $2,9 \%$ & $3,6 \%$ & $4,7 \%$ \\
5 & $0,6 \%$ & $2,0 \%$ & $2,5 \%$ & $2,5 \%$ & $3,9 \%$ & $6,2 \%$ & $5,4 \%$ & $6,2 \%$ \\
\hline
\end{tabular}

Fuente: Encuestas de Hogares del Programa MECOVI.

Elaboración: Propia.

La Tabla 7 presenta el ejercicio anterior, sólo que para la región urbana del país, ya que el estudio econométrico se concentra en estas regiones. La tendencia observada a nivel nacional permanece a nivel urbano, de hecho, en el caso de las familias del quintil 5 dicha tendencia se incrementa. Aunque también se observa que las familias de estratos bajos de ingreso incrementaron su participación en el uso de gas natural.

\section{TABLA 7}

CONSUMO DE GAS NATURAL POR QUINTIL DE INGRESO - URBANO

\begin{tabular}{|c|c|c|c|c|c|c|c|c|}
\hline Quintil & 1999 & 2000 & 2001 & 2002 & $2003-2004$ & 2005 & 2006 & 2007 \\
\hline 1 & $1,1 \%$ & $0,6 \%$ & $0,8 \%$ & $1,1 \%$ & $1,0 \%$ & $2,2 \%$ & $1,8 \%$ & $4,0 \%$ \\
2 & $1,5 \%$ & $1,3 \%$ & $1,0 \%$ & $0,4 \%$ & $1,5 \%$ & $2,3 \%$ & $3,0 \%$ & $3,1 \%$ \\
3 & $0,6 \%$ & $0,8 \%$ & $0,4 \%$ & $0,4 \%$ & $0,9 \%$ & $3,1 \%$ & $3,0 \%$ & $4,0 \%$ \\
4 & $1,5 \%$ & $1,0 \%$ & $1,4 \%$ & $1,5 \%$ & $1,8 \%$ & $4,5 \%$ & $5,0 \%$ & $7,4 \%$ \\
5 & $0,6 \%$ & $2,5 \%$ & $3,5 \%$ & $3,1 \%$ & $5,6 \%$ & $7,1 \%$ & $7,4 \%$ & $7,8 \%$ \\
\hline
\end{tabular}

Fuente: Encuestas de Hogares del Programa MECOVI.

Elaboración: Propia. 
Por su parte, la Tabla 8 presenta el consumo promedio familiar, expresado en unidades de energía, de gas natural de acuerdo a cada quintil de ingreso. En ella se observa que a medida que se incrementa el ingreso del hogar también lo hace el consumo de este producto, de hecho, el quintil superior de ingreso tiende a consumir el doble que el quintil inferior. Por su parte, la Tabla 9 presenta indicadores similares, sólo que para el caso del GLP. Contrastando estas dos tablas se confirma que el consumo de gas natural, en promedio, tiende a ser mayor al del GLP, manteniéndose en ambos casos la característica central de que mayor ingreso redunda en mayor consumo de energía.

TABLA 8

CONSUMO FAMILIAR PROMEDIO DE GAS NATURAL (MM BTU/MES)

\begin{tabular}{|c|c|c|c|c|c|c|c|c|}
\hline Quintil & 1999 & 2000 & 2001 & 2002 & $2003-2004$ & 2005 & 2006 & 2007 \\
\hline 1 & 0,762 & 0,834 & 0,651 & 0,837 & 0,938 & 0,548 & 0,806 & 0,691 \\
2 & 1,025 & 1,156 & 0,871 & 0,893 & 0,808 & 0,681 & 1,078 & 0,792 \\
3 & 1,572 & 0,976 & 0,876 & 1,024 & 0,907 & 0,801 & 0,784 & 0,763 \\
4 & 1,483 & 1,399 & 1,441 & 1,130 & 0,878 & 0,698 & 0,882 & 0,877 \\
5 & 1,757 & 1,453 & 2,104 & 1,374 & 1,281 & 1,358 & 1,153 & 1,134 \\
\hline
\end{tabular}

TABLA 9

CONSUMO FAMILIAR PROMEDIO DE GLP (MM BTU/MES)

\begin{tabular}{|c|c|c|c|c|c|c|c|c|}
\hline Quintil & 1999 & 2000 & 2001 & 2002 & $2003-2004$ & 2005 & 2006 & 2007 \\
\hline 1 & 0,537 & 0,521 & 0,493 & 0,482 & 0,531 & 0,451 & 0,489 & 0,493 \\
2 & 0,682 & 0,606 & 0,575 & 0,547 & 0,594 & 0,551 & 0,530 & 0,557 \\
3 & 0,738 & 0,638 & 0,638 & 0,607 & 0,655 & 0,579 & 0,584 & 0,601 \\
4 & 0,811 & 0,770 & 0,725 & 0,714 & 0,701 & 0,643 & 0,664 & 0,648 \\
5 & 0,928 & 0,981 & 0,880 & 0,813 & 0,839 & 0,677 & 0,709 & 0,686 \\
\hline
\end{tabular}

Como corolario de esta sección, es interesante contrastar los resultados de las encuestas de hogares con las estadísticas publicadas por la Superintendencia de Hidrocarburos. Esta institución gubernamental regulatoria publica, entre otra información, el número de hogares en Bolivia que posee instalada la conexión a la red secundaria de distribución. El resumen de estos datos se encuentra en la Tabla 10, en ella se encuentra el porcentaje de familias que poseen acceso al gas natural en la región urbana boliviana. 


\section{TABLA 10}

PORCENTAJE DE FAMILIAS URBANAS CON ACCESO A LA RED DE GAS NATURAL

\begin{tabular}{|l|c|c|c|c|r|r|r|r|}
\hline \multicolumn{1}{|c|}{ Departamento } & 1999 & 2000 & 2001 & 2002 & \multicolumn{1}{|c|}{2004} & \multicolumn{1}{c|}{2005} & \multicolumn{1}{c|}{2006} & \multicolumn{1}{c|}{2007} \\
\hline La Paz & $0,2 \%$ & $0,2 \%$ & $0,3 \%$ & $0,4 \%$ & $1,9 \%$ & $1,9 \%$ & $1,9 \%$ & $3,5 \%$ \\
Oruro & $0,1 \%$ & $0,2 \%$ & $0,2 \%$ & $0,4 \%$ & $7,5 \%$ & $7,6 \%$ & $7,7 \%$ & $13,8 \%$ \\
Potosí & $0,2 \%$ & $0,4 \%$ & $0,7 \%$ & $1,2 \%$ & $14,3 \%$ & $15,3 \%$ & $14,9 \%$ & $19,0 \%$ \\
Santa Cruz de la Sierra & $0,3 \%$ & $0,3 \%$ & $0,4 \%$ & $0,5 \%$ & $1,2 \%$ & $1,5 \%$ & $1,8 \%$ & $1,7 \%$ \\
Cochabamba & $1,2 \%$ & $2,7 \%$ & $3,8 \%$ & $5,1 \%$ & $6,4 \%$ & $8,1 \%$ & $8,6 \%$ & $9,5 \%$ \\
Chuquisaca & $2,5 \%$ & $3,2 \%$ & $3,5 \%$ & $3,5 \%$ & $3,4 \%$ & $4,4 \%$ & $6,8 \%$ & $10,2 \%$ \\
Tarija & $7,1 \%$ & $7,2 \%$ & $8,2 \%$ & $8,8 \%$ & $23,6 \%$ & $35,8 \%$ & $45,3 \%$ & $64,9 \%$ \\
\hline Total & $0,7 \%$ & $1,0 \%$ & $1,3 \%$ & $1,6 \%$ & $4,0 \%$ & $4,9 \%$ & $5,6 \%$ & $7,8 \%$ \\
\hline
\end{tabular}

Fuente: Encuesta de Hogares - MECOVI.

Elaboración: Propia a partir de los datos del Censo 2001, sobre número de hogares y tasa de crecimiento urbana intersencal 1992-2001.

El contraste entre los resultados de las encuestas de hogares y los publicados por la Superintendencia de Hidrocarburos se encuentra en la Figura 1, de ella se desprenden algunos comentarios: 1) es positivo advertir la consistencia de la información, los resultados de las encuestas de hogares son compatibles con la información de la Superintendencia de Hidrocarburos; ${ }^{6} 2$ ) a partir del año 2004, la tasa de crecimiento del número de instalaciones es superior a la tasa de crecimiento en el consumo de gas natural; de alguna manera este resultado refleja que la agresiva política de expansión en las redes de distribución de gas natural en Bolivia no siempre estuvo acompañada de sistemas que incentiven al uso de este producto; finalmente, 3) aún el acceso a gas natural en Bolivia es muy pequeño, hasta el año 2006 menos del 8\% de familias tenía acceso a este producto y algo más del $5 \%$ lo utiliza como insumo en el hogar.

\section{Precios}

El presente estudio de la demanda por gas natural está vinculado al análisis del consumo de GLP, toda vez que ambos productos, se presume, son sustitutos. En este sentido, en esta sección se presentará el comportamiento de los precios de estos productos: 1) gas natural; 2) GLP, y 3) otros bienes y servicios.

La Tabla 11 presenta el resumen de precios durante el período 1999-2007. Es útil recordar que las encuestas de hogares se realizan en noviembre de cada año, por ello, los precios utilizados son el promedio de este mes. Por otra parte, dado que durante el período 2003-2004 se hizo una encuesta continua, los precios de ese período son los promedios simples entre noviembre de 2003 y noviembre de 2004.

6 Las encuestas de hogares se construyen en base a muestras poblacionales; por otro lado, la información que posee la Superintendencia de Hidrocarburos es reportada por las empresas distribuidoras de gas natural. 


\section{FIGURA 1}

ACCESO Y USO DEL GAS NATURAL EN EL AREA URBANA

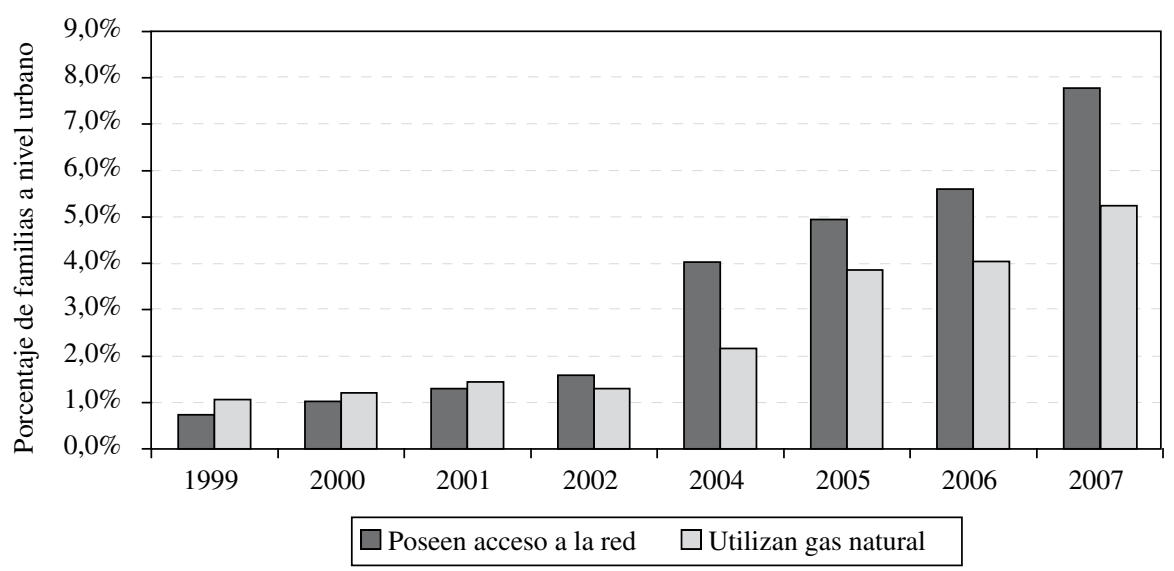

Los precios finales del gas natural distribuido por redes expresados en US $\$ / M C^{7}$ no cambiaron durante el período de análisis, por esta razón, las variaciones observadas en la tabla anterior son el resultado de la devaluación de la moneda nacional contra el dólar americano. En el caso particular de las tarifas aplicadas por YPFB, ellas se mantuvieron constantes en moneda nacional, Bs./MPC 22,22, esta política fue definida en reuniones de directorio y es completamente válida, toda vez que el marco legal establecía precios tope.

Es útil señalar que desde el año 1992 no existió una metodología clara para fijar los precios al consumidor final del gas natural. Hubo varios intentos por establecer dicha metodología en el pasado, sin embargo, ellos no tuvieron éxito. En este sentido, se puede afirmar que estos precios no se ajustaron por incrementos/decrementos en los costos de operación y/o la rentabilidad de los operadores. Por ello, surge la hipótesis de que el costo de capital de las nuevas instalaciones de gas natural deban ser subsidiadas por la empresa estatal de hidrocarburos (YPFB), dado que con alta probabilidad el precio final de este producto permanecerá inalterado.

El precio del GLP ha tenido pocas modificaciones, ya que fue (y es) política de Estado no modificarlo aun cuando los precios internacionales se incrementen de forma importante, de ahí surge la idea del "subsidio al precio del GLP”. Finalmente, el precio de los otros bienes y servicios fue estimado a partir del Indice de Precios al Consumidor (IPC) calculado por el INE, a este índice se le restó la variación de precios de los combustibles utilizados en la cocina.

$7 \quad$ MPC = Millar de pie cúbico. 


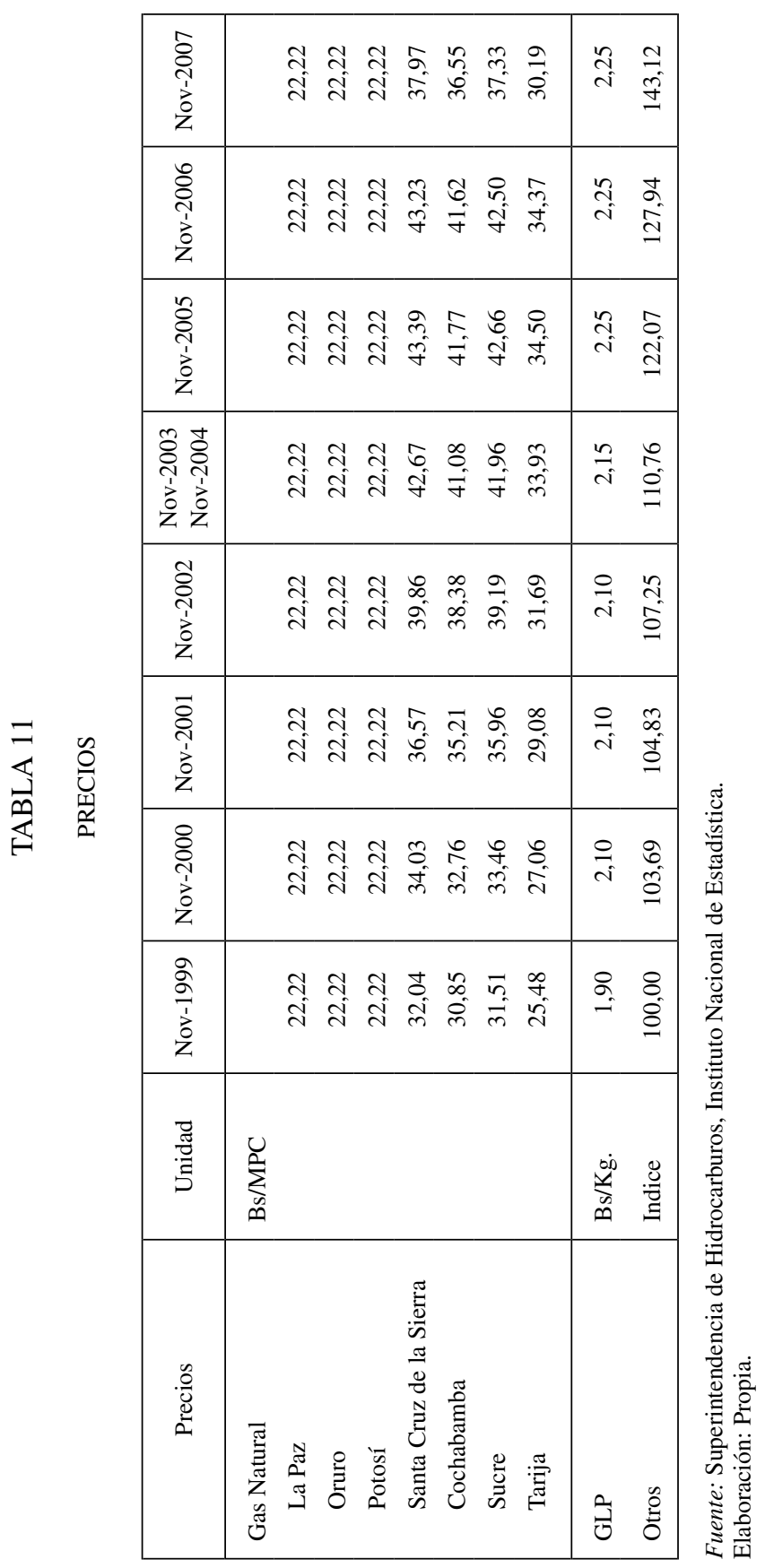


Dado que la tasa de devaluación fue menor a la tasa de inflación, entonces los precios del gas natural distribuido a través de redes disminuyeron considerablemente. Una mirada detallada de los datos muestra que a partir del año 2004 el precio relati$v^{8}$ del gas natural comenzó a disminuir, manteniéndose la brecha entre Tarija, el eje occidental y el resto de departamentos, ver Figura 2.

\section{FIGURA 2}

PRECIOS DEL GAS NATURAL EXPRESADOS EN BS. 1999

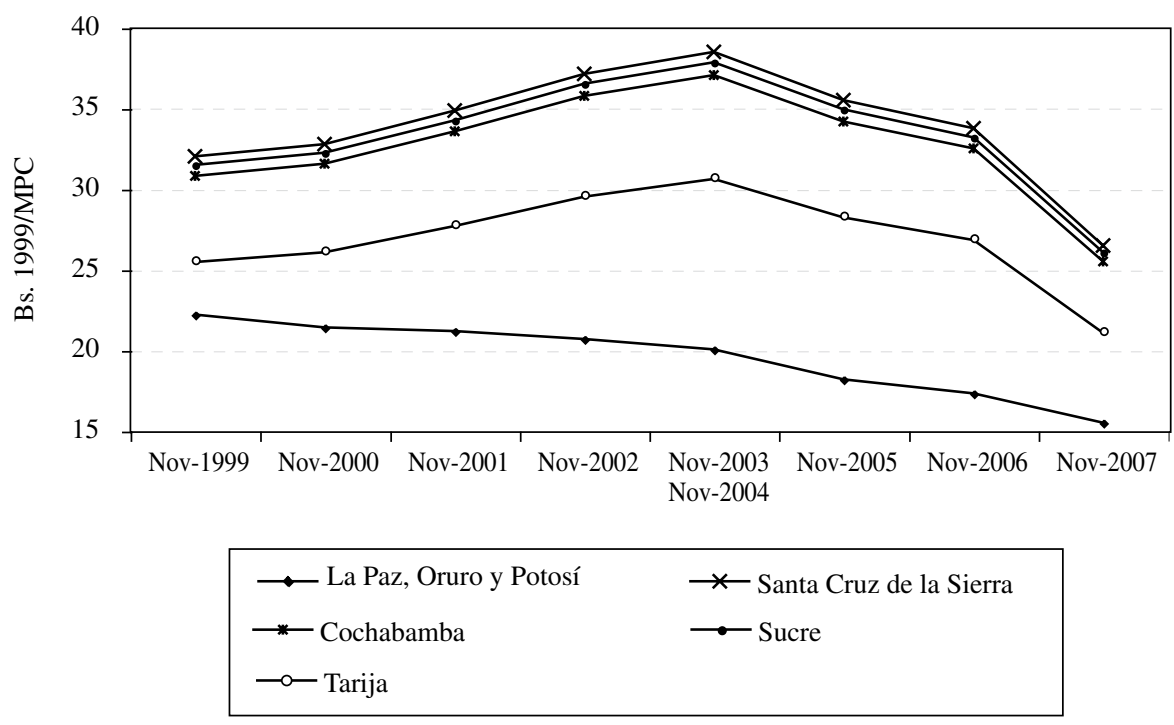

Por su parte, el precio relativo del GLP también disminuyó; la denominada "política de precios subsidiados" ocasiona que el precio real de este producto presente el comportamiento expresado en la Figura 3. De hecho, existe la conjetura de que esta política de precios subsidiados afectaría negativamente el consumo de gas natural, dado que el precio por unidad calorífica es menor. Por ello, dentro las políticas energéticas que proponen los gobiernos de turno, se encuentra el incentivar el consumo de gas natural "eliminando el subsidio al precio del GLP", es decir, incrementando el precio del GLP a un precio "paridad de exportación".

8 Este precio, como también el del GLP, fue deflactado por el índice calculado para el tercer grupo de bienes. 
FIGURA 3

PRECIOS DEL GLP EXPRESADOS EN BS. 1999

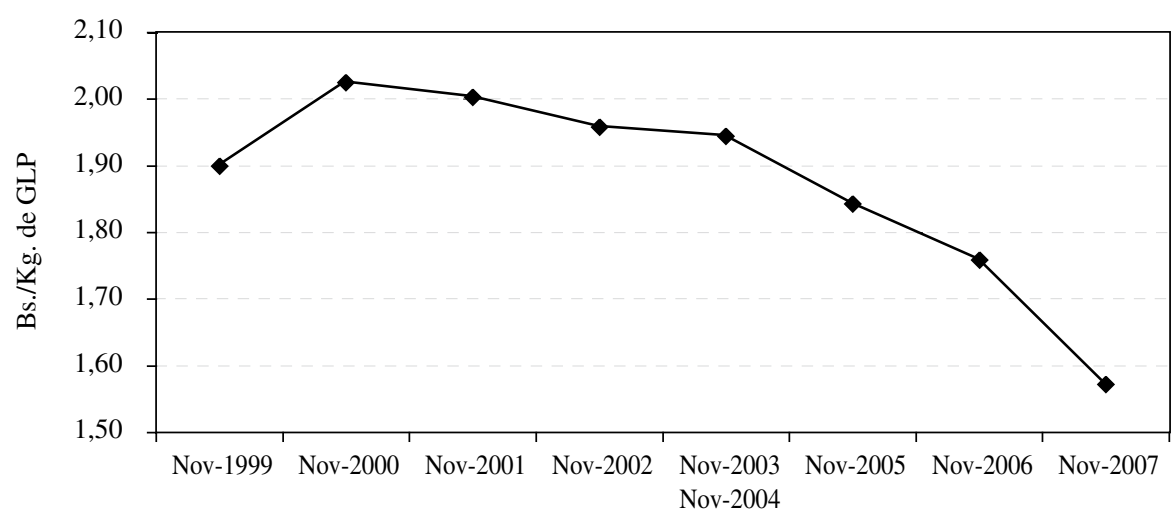

UN MODELO CASI IDEAL DE DEMANDA

La disponibilidad de información acerca de las características de consumo de las familias (inserta en encuestas de hogares) y la posibilidad de realizar estimaciones econométricas a gran escala con un costo relativamente bajo ocasionaron que en los últimos años se presente un renovado interés en la estimación de funciones de demanda por aquellos bienes consumidos por unidades familiares.

Desde su publicación en 1980, el Sistema Casi Ideal de Demanda (AIDS por su sigla en inglés) propuesto por Deaton y Muellbauer ${ }^{9}$ se utilizó en estimaciones de funciones de demanda por variados bienes. ${ }^{10}$ Esta metodología resulta bastante atractiva porque además de utilizar información disponible en las encuestas de hogares (no siempre utilizada al momento de estimar una función de demanda) proporciona relaciones "teóricamente adecuadas" en función a los datos de fuente primaria.

Aplicaciones de esta metodología a la demanda de energía a nivel residencial se encuentran en Labandeira y López (2002), Labandeira et al. (2004) y Labandeira et al. (2005). En estos trabajos se observa que dicha metodología funciona muy bien para predecir los efectos de corto plazo que tendrían algunas políticas energéticas, por ejemplo, variaciones en los precios al consumidor final.

Dentro de las modificaciones propuestas al modelo original, la realizada por Banks et al. (1997) resultó bastante atractiva y por ello ampliamente utilizada. Los autores sugieren que es necesario verificar la presencia de relaciones cuadráticas entre el gasto total de las familias y el incurrido en determinado bien. En este sentido, para verificar si la propuesta original es suficiente o no para lograr una estimación más precisa, a

9 Deaton y Muellbauer (1980).

10 Para un detalle interesante de su uso ver García (2003). 
continuación se analiza el comportamiento del gasto en gas natural y GLP, en función al gasto total (como aproximación del ingreso permanente) del hogar.

La Figura 4 presenta los resultados de la estimación no paramétrica del tipo kernel realizada entre el gasto en gas natural y el gasto total, en todas aquellas observaciones donde el gasto en este bien es positivo. Ciertamente la relación es negativa, mayor gasto por parte de la familia implica una participación menor del gas natural. Por otra parte, la Figura 5 presenta los resultados de un ejercicio numérico similar, sólo que para el caso del GLP también se advierte una relación negativa en ambas variables. De la inspección visual no queda claro que la relación entre estas dos variables sea lineal, por esta razón es necesario realizar algún análisis econométrico adicional.

\section{FIGURA 4}

PARTICIPACION DEL GASTO EN GAS NATURAL

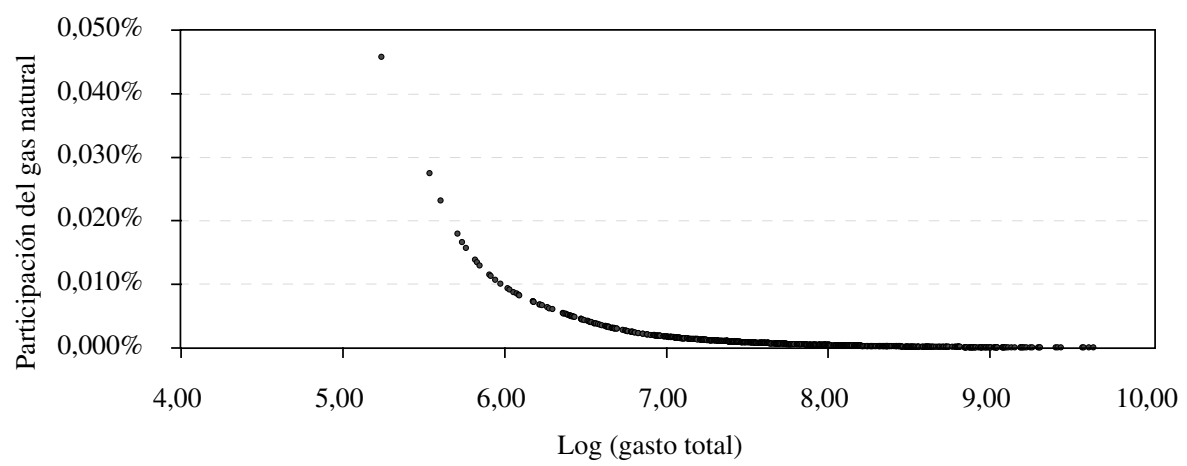

FIGURA 5

PARTICIPACION DEL GASTO EN GLP

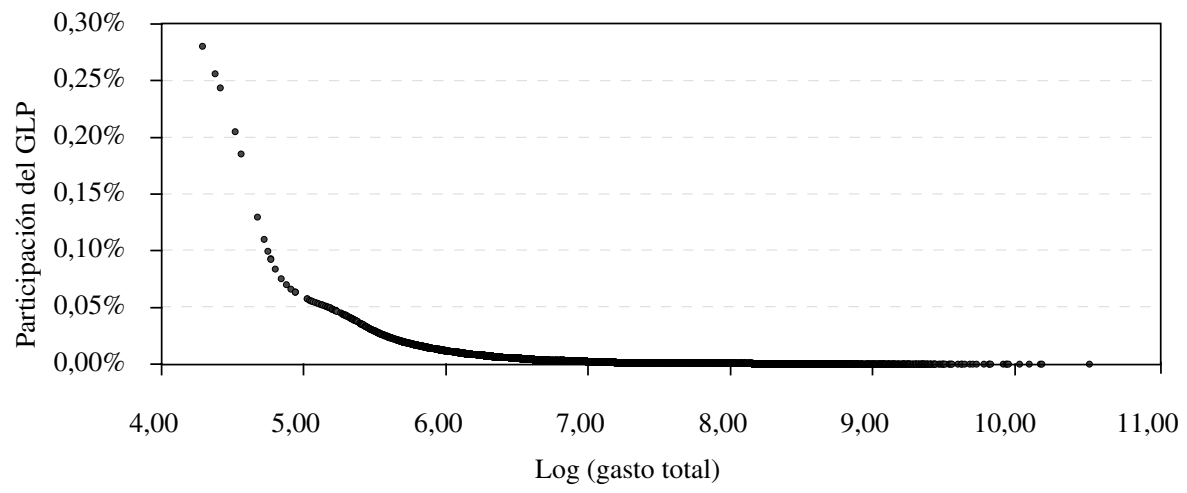


La Tabla 12 presenta las estimaciones entre la participación en el gasto y el logaritmo del gasto total $(m)$ y el logaritmo del gasto total al cuadrado $\left(\mathrm{m}^{2}\right)$, utilizando $\mathrm{MCO}^{11}$ y $\mathrm{MCO}$ extendido (Wu-Hausman). ${ }^{12}$ En ambas estimaciones se advierte que el término cuadrático es significativo, tanto para el gas natural como para el GLP. ${ }^{13}$ Por esta razón, en lo que sigue de esta sección se describirá el modelo QUAIDS, su derivación y estimación, dado que será éste el utilizado en la sección empírica.

TABLA 12

CURVAS DE ENGEL

\begin{tabular}{|c|c|c|c|c|}
\hline \multirow[b]{2}{*}{ Variable } & \multicolumn{2}{|c|}{$\mathrm{MCO}$} & \multicolumn{2}{|c|}{ Wu-Hausman } \\
\hline & $\begin{array}{c}\text { Gas } \\
\text { Natural }\end{array}$ & GLP & $\begin{array}{c}\text { Gas } \\
\text { Natural }\end{array}$ & GLP \\
\hline Constante & 13,956 & 1,163 & 13,422 & 1,237 \\
\hline \multirow{2}{*}{ Vivienda propia $(1=$ Sí, 0 = No $)$} & 0,071 & 0,080 & 0,074 & 0,081 \\
\hline & 0,028 & 0,004 & 0,028 & 0,004 \\
\hline \multirow[t]{2}{*}{ Número miembros familia } & 0,051 & 0,059 & 0,051 & 0,059 \\
\hline & 0,007 & 0,001 & 0,007 & 0,001 \\
\hline \multirow[t]{2}{*}{ Número de cocinas en el hogar } & 0,147 & $-0,003$ & 0,144 & $-0,005$ \\
\hline & 0,046 & 0,007 & 0,046 & 0,007 \\
\hline Número de hornos en el hogar & $\begin{array}{l}\mathbf{0 , 0 5 1} \\
0,031\end{array}$ & $\begin{array}{r}\mathbf{- 0 , 0 2 6} \\
0,006\end{array}$ & $\begin{array}{l}\mathbf{0 , 0 6 4} \\
0,032\end{array}$ & $\begin{array}{r}\mathbf{- 0 , 0 2 6} \\
0,006\end{array}$ \\
\hline \multirow[t]{2}{*}{ Número de bienes que utilizan energía eléctrica } & 0,010 & 0,005 & 0,007 & 0,006 \\
\hline & 0,006 & 0,001 & 0,006 & 0,001 \\
\hline \multirow[t]{2}{*}{ Variable ficticia Distribuidoras Privadas } & 0,392 & $-0,153$ & 0,394 & $-0,159$ \\
\hline & 0,071 & 0,033 & 0,071 & 0,033 \\
\hline \multirow[t]{2}{*}{ Log precio gas natural } & $-0,704$ & 0,340 & $-0,700$ & $\mathbf{0 , 3 5 1}$ \\
\hline & 0,156 & 0,063 & 0,161 & 0,063 \\
\hline \multirow[t]{2}{*}{ Log precio GLP } & 2,921 & $-\mathbf{0 , 2 3 0}$ & 2,871 & $-0,255$ \\
\hline & 0,495 & 0,096 & 0,501 & 0,096 \\
\hline Log (gasto total) & $\begin{array}{r}-3,841 \\
0364\end{array}$ & $\begin{array}{r}-\mathbf{0 , 8 2 9} \\
0,050\end{array}$ & $\begin{array}{r}-\mathbf{3 , 7 3 5} \\
0367\end{array}$ & $\mathbf{- 0 , 8 5 5}$ \\
\hline \multirow[t]{2}{*}{ Log (gasto total)2 } & 0,185 & $-\mathbf{0 , 0 0 6}$ & $\mathbf{0 , 1 8 1}$ & $-0,004$ \\
\hline & 0,022 & 0,003 & 0,022 & 0,003 \\
\hline \multirow[t]{2}{*}{$\mathbf{v}$} & - & - & $-0,055$ & $-0,005$ \\
\hline & - & - & 0,044 & 0,006 \\
\hline R2 & 0,46 & 0,686 & 0,47 & 0,68 \\
\hline R2 ajustado & 0,46 & 0,686 & 0,46 & 0,68 \\
\hline $\mathrm{F}$ & 161,17 & 7.402 & 141,31 & $6.618,12$ \\
\hline
\end{tabular}

$v$ es el término de error al instrumentar el gasto con el ingreso.

11 La estimación utilizó MCO ponderados por el factor de expansión.

12 Esta última incluye el error de la regresión entre el logaritmo del gasto total y el logaritmo del ingreso, logaritmo de precios relativos, número de miembros en el hogar, pertenencia al eje central de Bolivia y una variable de tendencia.

13 En cada una de las muestras se utilizaron aquellas observaciones que reportan un gasto positivo en el energético en cuestión. 
El QUAIDS se deriva de la siguiente especificación logarítmica cuadrática de la función de gasto de un determinado consumidor: ${ }^{14}$

$$
\ln c(u, p)=\ln a\left(p_{t}\right)+\frac{u \cdot \ln b\left(p_{t}\right)}{1-u \cdot g\left(p_{t}\right)}
$$

Donde:

$u$ es el nivel de utilidad que puede variar entre 0 (subsistencia) y 1 (riqueza). ${ }^{15}$ $a(p)$ es el costo de subsistencia y $b(p)$ es el costo de la riqueza.

A continuación se presentan las funciones de los índices de precios considerados anteriormente:

$$
\begin{gathered}
\ln a\left(p_{t}\right)=\alpha_{0}+\sum_{i=1}^{n} \alpha_{i} \cdot \ln p_{i, t}+\frac{1}{2} \cdot \sum_{i=1}^{n} \sum_{j=1}^{n} \gamma_{i, j} \cdot \ln p_{i, t} \cdot \ln p_{j, t} \\
b\left(p_{t}\right)=\prod_{i=1}^{n} p_{i, t}^{\beta_{i}} \\
g\left(p_{t}\right)=\sum_{i=1}^{n} \lambda_{i} \cdot \ln p_{i, t}
\end{gathered}
$$

Donde:

$$
\alpha_{i}=\alpha_{i, 0}+\sum_{k=1}^{K} \alpha_{i, k} \cdot z_{k}^{\alpha}
$$

Las variables $\mathrm{z}$ denotan características demográficas como educación del jefe de hogar, vivienda propia, etc. Combinando las ecuaciones anteriores se obtiene la siguiente forma funcional de la ecuación de demanda a estimarse por cada bien: ${ }^{16}$

14 Una función de gasto define el gasto mínimo que requiere el consumidor para alcanzar determinado nivel de utilidad con precios dados; en el modelo inicial desarrollado por Deaton y Muellbauer (1980) la función de gasto tomaba la siguiente forma: $\ln c\left(u, p_{t}\right)=(1-u) \cdot \ln a\left(p_{t}\right)+u \cdot \ln b\left(p_{t}\right)$. Se aprecia claramente la razón por la que los índices de precios $\mathrm{a}(\mathrm{p})$ y b(p) corresponden a determinados estados de bienestar; sin embargo, su versión cuadrática propuesta por Banks et al. (1997), permite que las curvas de Engel resultantes no sólo sean lineales, sino también cuadráticas.

15 La traducción no es del todo correcta, la palabra original en inglés es bliss.

16 Ver Anexo 1. 


$$
w_{i, t}=\alpha_{i}+\sum_{j=1}^{n} \gamma_{i, j} \cdot \ln p_{j, t}+\beta_{i} \cdot \ln \left[\frac{m_{t}}{a\left(p_{t}\right)}\right]+\frac{\lambda_{i}}{b\left(p_{t}\right)} \cdot \ln \left[\frac{m_{t}}{a\left(p_{t}\right)}\right]^{2}+\varepsilon_{i, t}
$$

Donde $w_{i, t}$ es la participación en el gasto total del bien $i$ en el período t, $m_{t}$ es el ingreso total (que puede estar medido como el gasto total) en el mismo período, para determinada familia. Resaltan dos características de la ecuación (5); la primera es la relación que existe entre la demanda del bien $i$ no sólo con su propio precio, sino también con el precio del resto de bienes considerados; la segunda, es la relación cuadrática entre la demanda del bien y el ingreso total, esta forma funcional determina que las curvas de Engel sean no lineales.

También es posible obtener, a partir de esta forma funcional, las elasticidades ingreso y precio de cada uno de los bienes. Diferenciando la ecuación (5) respecto al ingreso (gasto total) se obtiene la elasticidad ingreso del bien $i$ para cada familia:

$$
e_{i}=1+\frac{\mu_{i}}{w_{i}}
$$

Donde:

$$
\mu_{i}=\beta_{i}+\left(2 \cdot \lambda_{i} \cdot \ln \left(\frac{m_{t}}{a\left(p_{t}\right)}\right)\right) \cdot \frac{1}{b\left(p_{t}\right)}
$$

Diferenciando la ecuación (5) respecto del precio del bien $j$ es posible obtener la elasticidad precio no compensada de este bien:

$$
e_{i j}^{u}=\frac{\mu_{i j}}{w_{i}}-\delta_{i j}
$$

Donde:

$$
\mu_{i j}=\gamma_{i j}-\mu_{i} \cdot\left[\alpha_{j}+\sum_{k=1}^{n} \gamma_{j k} \cdot \ln p_{k}\right]-\left[\beta_{j} \cdot \lambda_{i}\left(\ln \frac{m}{a(p)}\right)^{2}\right] \cdot \frac{1}{b(p)}
$$

$\delta_{i j}=0$ si $i \neq j$ y $\delta_{i j}=1$ si $i=j$.

Es interesante notar que, de acuerdo a la forma cómo se calculan estas elasticidades, es posible obtener, para cada familia estudiada, la respuesta de la cantidad consumida ante cambios en el nivel de precios o ingreso.

Las técnicas de estimación econométrica del sistema definido por las ecuaciones (2), (3) y (5) varían ampliamente, desde estimaciones de máxima verosimilitud hasta procesos iterativos de regresiones mínimo cuadráticas. En este documento se utilizará 
aquella propuesta por Blundell y Robin (1999), que consiste en estimar la ecuación (5) a través del siguiente proceso iterativo: se aproximan las ecuaciones (2) y (3) con el Indice de Stone ${ }^{17}$ y $b\left(p_{t}\right)=1$, con estos valores, a través de mínimos cuadráticos, se estima la ecuación (5); luego con los valores estimados se aproximan nuevamente las ecuaciones (2) y (3) y el proceso iterativo continúa hasta que determinada convergencia es alcanzada en los parámetros estimados.

Debido a la presencia de correlación contemporánea entre los errores y el ingreso total (a partir de este momento, gasto total), la técnica de mínimos cuadráticos produce estimadores inconsistentes, por esta razón es necesario instrumentar el gasto total con el ingreso total, el cual, bajo condiciones de separabilidad, debería estar no correlacionado con el error. También se deben tomar en cuenta algunas restricciones teóricas de simetría y homogeneidad de grado cero. La condición de homogeneidad se impone utilizando precios relativos y la condición de simetría $\gamma_{i j}=\gamma_{j i}$ es impuesta durante la estimación.

Finalmente, dado que muchas familias no reportan gasto en gas natural se utilizó la metodología propuesta en Gómez (1996). Esta sugiere que en esta situación se estime la variable $\lambda$ a través de la técnica de dos etapas propuesta por Heckman. Entonces, en la primera etapa se estima la decisión de utilizar gas natural a través de un modelo probit, luego, con los resultados de dicha estimación, se crea una nueva variable $\lambda$ que es añadida en (5) como un regresor adicional.

\section{RESULTADOS}

En la estimación del modelo QUAIDS se utilizaron tres categorías de gasto: gas natural, GLP y otros. ${ }^{18}$ Para cumplir con el supuesto de homogeneidad, no se estimó la demanda para la categoría "otros", por ello, su precio fue utilizado como numerario. Se estimaron dos ecuaciones de demanda cuya forma funcional se especifica en (5), la variable $m_{t}$ se obtuvo del gasto total, los precios relativos $p_{j, t}$ fueron calculados para el gas natural y GLP; finalmente, la participación del gasto se obtuvo de las encuestas de hogares del período 1999-2006.

Por su sencillez y robustez de los resultados la metodología de estimación es a través del método de mínimos cuadrados iterativos propuesto por Blundell y Robin (1999); 19 los resultados, tanto de la estimación del modelo QUAIDS como de la variable $\lambda$, se encuentran en el Anexo 2. A continuación sólo se presentan las elasticidades precio e ingreso de cada uno de los bienes estimados (Tabla 13) ${ }^{20}$ El signo y la magnitud de las elasticidades son los esperados. Los resultados sugieren que, en promedio,

\footnotetext{
17 El Indice de Stone se define como $\ln p_{t}^{*}=\sum_{j=1}^{n} w_{j, t} \ln \left(p_{j, t}\right)$.

18 En toda la muestra el 9,2\% de las familias no gasta ni en gas natural ni en GLP.

19 Para revisar aplicaciones de esta metodología ver Blow (2003), Labandeira et al. (2005) y Banks et al. (1997).

20 Valores promedio ponderados para toda la muestra, siguiendo a Banks et al. (1997) el ponderador utilizado es la participación observada del gasto en este producto, respecto del gasto total.
} 
la demanda de gas natural es más sensible a variaciones en el precio e ingreso, que en el caso del GLP. La tabla también presenta los resultados de una especificación alternativa (tradicional) de la función de demanda ${ }^{21}$ donde la cantidad demandada del energético depende de los precios, el ingreso y determinadas características del hogar. Con excepción de la elasticidad ingreso del gas natural, los resultados son muy similares, insinuando su robustez.

TABLA 13

ELASTICIDADES PRECIO E INGRESO

\begin{tabular}{|l|c|c|c|c|}
\hline \multirow{2}{*}{ Metodología } & \multicolumn{2}{|c|}{ Elasticidad gas natural } & \multicolumn{2}{c|}{ Elasticidad GLP } \\
\cline { 2 - 5 } & Precio & Ingreso & Precio & Ingreso \\
\hline QUAIDS & $-0,99991$ & 0,52848 & $-0,49495$ & 0,07917 \\
Alternativa & $-1,00586$ & 0,18792 & $-0,49230$ & 0,07905 \\
\hline
\end{tabular}

Elaboración: Propia.

La versatilidad de la especificación QUAIDS radica en que es posible desagregar los resultados a nivel familiar, en este sentido, a continuación se presentarán las elasticidades precio e ingreso, de acuerdo a cada quintil de ingreso. ${ }^{22}$ Los resultados sugieren varias e interesantes conjeturas, la primera se refiere a la elasticidad precio de ambos bienes; se observa que a medida que el ingreso familiar se incrementa, también lo hace esta elasticidad en el caso del gas natural (o permanece constante en algunos casos), sin embargo, en el caso del GLP, este indicador disminuye. ¿Por qué la sensibilidad es mayor en el caso del gas natural? Una posible hipótesis es que el mercado de gas natural en Bolivia aún es emergente, por ello, variaciones en el precio de este producto generan un mayor consumo, dado que las familias están en proceso de reconversión de su portafolio de bienes.

La diferencia entre los dos producto, en el caso de las elasticidades ingreso, es evidente, mientras que las familias de mayor poder adquisitivo tienden a considerar al GLP como un bien inferior, ${ }^{23}$ en el caso del gas natural dicha elasticidad es positiva. De esta forma, los resultados sugieren dos posibilidades que no son excluyentes: 1) a mayor ingreso menor consumo de GLP, posiblemente porque el hogar utiliza energías alternativas y; 2) el incremento en el consumo de gas natural es proporcionalmente mayor a medida que el ingreso familiar sube, posiblemente ello se debe a que son las familias de mayor ingreso quienes pueden, en el corto plazo, reconvertir su portafolio

21 Ver especificación y resultados en el Anexo 3.

22 La aproximación de la variable ingreso es el gasto total.

23 Resultado similar se encuentra en Labandeira et al. (2005). 
de bienes durables, generado así un mayor consumo respecto a familias más pobres, que no tienen esta posibilidad inmediata. Este último resultado es consistente con aquellos observados en las tablas de consumo energético anteriores.

TABLA 14

ELASTICIDADES POR QUINTIL DE INGRESO

\begin{tabular}{|c|c|c|c|c|}
\hline \multirow{2}{*}{ Quintil } & \multicolumn{2}{|c|}{ Elasticidad gas natural } & \multicolumn{2}{c|}{ Elasticidad GLP } \\
\cline { 2 - 5 } & Precio & Ingreso & Precio & Ingreso \\
\hline 1 & $-1,00024$ & 0,66528 & $-0,84758$ & 0,45682 \\
2 & $-1,00010$ & 0,70884 & $-0,77109$ & 0,35211 \\
3 & $-1,00006$ & 0,54141 & $-0,70235$ & 0,27604 \\
4 & $-1,00003$ & 0,34457 & $-0,58573$ & 0,15926 \\
5 & $-0,99978$ & 0,18181 & 0,21915 & $-0,53009$ \\
\hline
\end{tabular}

Elaboración: Propia.

Gracias a la metodología utilizada, también es posible estimar el cambio en el bienestar de cada familia, ante variaciones en los precios finales del gas natural y GLP. Por esta razón, en la siguiente sección se estudiará el impacto sobre la utilidad de las familias, de políticas energéticas que alteren el precio relativo de ambos bienes.

\section{ANALISIS DE BIENESTAR}

Una de las propuestas preferidas dentro el sector gubernamental boliviano es la disminución del precio del gas natural consumido por el sector residencial, tal como se presentó en la introducción de este documento. En este sentido, esta sección analiza el cambio en el bienestar de las familias ante una disminución en el precio del gas natural distribuido por redes a Bs. 8 por metro cúbico para todos los consumidores.

La metodología utilizada es la propuesta por West y Williams III (2002), quienes proponen estudiar el impacto sobre el bienestar bajo tres supuestos: 1) que no existe reacción por parte de las familias ante cambios en el precio con elasticidades precio, propias y cruzadas, iguales a cero; 2) considerando solamente las elasticidades precio propias y; 3 ) considerar las elasticidades precio propias y cruzadas. Bajo el primer supuesto, el cambio en el bienestar es simplemente la variación en el gasto total de cada familia, manteniendo constante la cantidad demandada del energético. En la segunda metodología el cambio en el gasto de cada familia $k$ viene dado por la siguiente especificación: 


$$
\Delta G=\sum_{i}\left\{\frac{x_{i}^{k} \bar{p}_{i}^{k}}{e_{i}^{k}+1}\left[1-\left(\frac{p_{i}^{k}}{\bar{p}_{i}^{k}}\right)^{e_{i}^{k}+1}\right]\right\}
$$

Donde:

$x_{i}^{k}=$ Demanda de gas natural expresada en unidades volumétricas.

$\bar{p}=$ Vector de precios previa a la reforma.

$p=$ Vector de precios post reforma.

$e_{i}^{k}=$ Elasticidad precio compensada propia.

Finalmente, cuando sí se consideran las elasticidades precio cruzadas, la metodología QUAIDS permite construir la variación equivalente $(V E)$ a partir de la función indirecta de utilidad de las familias, a través de la siguiente expresión:

$$
V E=\bar{m} e^{\left\{\beta_{0} \prod_{k}^{-\beta_{k}}[V(m, p)-V(\bar{m}, \bar{p})]\right\}}-\bar{m}
$$

Donde:

$\bar{m} \quad=$ Gasto total antes de la reforma.

$m$ = Gasto total después de la reforma.

$V()=$. Función indirecta de utilidad.

La Tabla 15 presenta el cambio en el indicador de bienestar bajo cada uno de los tres métodos explicados anteriormente, agrupados según quintil de ingreso. Los resultados son de interpretación directa; cuando se asume que la demanda de las familias no se modificará, entonces el ahorro de las familias con la disminución en el precio del gas natural es mayor en el quintil de mayor ingreso. Es decir, disminuyendo el precio a los niveles señalados por el Gobierno boliviano, las familias del quintil 5 ahorrarían Bs. 47.723, mientras que las familias más pobres, ahorrarían Bs. 7.661. Cuando se permite cierta sensibilidad en la demanda de gas natural, el ahorro tiende a disminuir; la razón es clara, menor precio genera mayor consumo y por ello las familias gastan más en gas natural. Sin embargo, el carácter regresivo de la medida prevalece bajo las tres alternativas.

Tomando en cuenta los resultados encontrados, en particular aquel que señala que las familias de mayor ingreso tienden a consumir una cantidad mayor de gas natural, sería razonable proponer que dentro de la nueva estructura de precios del gas natural aplicada al sector residencial en Bolivia se incorporen precios no lineales, de tal forma, que familias con mayor consumo enfrenten precios mayores. Dado que los objetivos 
TABLA 15

CAMBIO EN LOS INDICADORES DE BIENESTAR

\begin{tabular}{|c|c|c|c|}
\hline Quintil & Método I & Método II & Método III \\
\hline 1 & $-7,661$ & $-2,135$ & $-5,409$ \\
2 & $-8,382$ & $-2,431$ & $-2,789$ \\
3 & $-14,155$ & $-4,125$ & $-3,961$ \\
4 & $-18,272$ & $-5,379$ & $-5,085$ \\
5 & $-47,723$ & $-11,433$ & $-8,460$ \\
\hline
\end{tabular}

Elaboración: Propia.

políticos muchas veces prevalecen sobre aquellos técnicos, entonces, no se puede desechar la idea de que en el futuro los precios del gas natural continuarán subsidiados o, dicho de otra forma, no cubrirán los costos de operación y capital pertinentes. Por ello, una estructura de precios no lineal podría disminuir la distorsión, cobrando el precio adecuado a familias de alto consumo y subsidiando a aquellas que demandan cantidades pequeñas de este producto.

\section{CONCLUSIONES}

El consumo de gas natural en Bolivia aún se encuentra en la primera etapa de desarrollo, de hecho, el consumo interno está concentrado en el sector industrial, aunque las tasas de crecimiento de consumo de gas natural vehicular y residencial no dejan de ser interesantes. En este contexto gran parte de la discusión sobre política energética está concentrada en hallar los instrumentos adecuados para fomentar el consumo de gas natural en el mercado interno, y en particular, beneficiar a la gente más pobre con dichas políticas.

Analizando las Encuestas de Hogares, periódicamente realizadas por el Instituto Nacional de Estadística en Bolivia, se advierte que el porcentaje de familias que utiliza gas natural se incrementó en los últimos años. Sin embargo, contrastando estas cifras con aquellas publicadas por la Superintendencia de Hidrocarburos, el crecimiento del consumo es menor al crecimiento de la expansión del sistema de distribución de gas natural. Sugiriendo de esta forma que la agresiva política de ampliación en las redes de distribución no siempre estuvo acompañada de otras complementarias que incentiven su uso.

Por otra parte, discriminando el consumo de gas natural, reportado en las encuestas de hogares, por quintil de ingreso, los resultados son regresivos. En el período 1999-2007, el uso del gas natural en las familias más ricas (quintil 5) se incrementó de $0,6 \%$ a $7,8 \%$; sin embargo, en el quintil 1 se observa un crecimiento de $1,1 \%$ a $4,0 \%$. Una posible explicación de este hecho se encuentra en la posibilidad de reconversión del portafolio de bienes dentro el hogar, ella es mayor en las familias de mayor ingreso. 
El objetivo central de este documento es conocer cuáles son las principales características del consumo de gas natural domiciliario. En particular, utilizando la información proveniente de las encuestas de hogares realizadas por el Instituto Nacional de Estadística de Bolivia, se aplica la metodología propuesta por Deaton y Muellbauer (1980), un Sistema Casi Ideal de Demanda, para estimar una relación funcional entre las variables sujetas de análisis. Se introduce además la modificación propuesta por Banks et al. (1997), que consiste en permitir relaciones cuadráticas entre el gasto en cada bien y el gasto total de cada familia.

Los resultados encontrados, en general, son los esperados, la elasticidad precio del gas natural y GLP es menor a la unidad y la elasticidad ingreso es positiva. Llama la atención que la elasticidad ingreso del quintil más alto del ingreso, en el caso del GLP, sea negativa. Por otra parte, con las estimaciones de demanda fue posible evaluar el impacto sobre el bienestar de las familias, de una disminución en el precio del gas natural. Esta simulación cobra relevancia porque es la favorita, en varios sectores de opinión, para incentivar el consumo de gas natural. Se advierte que disminuir el precio del gas natural beneficiaría en mayor proporción a familias ricas, bajo las tres metodologías utilizadas.

Ciertamente la principal limitación en este trabajo es la no disponibilidad de encuestas referidas únicamente al consumo final de energía por parte de las familias. La construcción de los modelos y los resultados encontrados, si bien son consistentes entre sí, deben ser confirmados y estimados nuevamente con nuevas encuestas exclusivas del sector energético. Aún así, los resultados no dejan de llamar la atención; curiosamente, las elevadas reservas de gas natural y el afán por incentivar su consumo no siempre generan políticas socialmente progresivas. Ya en el final, será bueno reflexionar sobre aquella anécdota de una familia pobre del Chaco boliviano, que sin tener los servicios de alcantarillado, agua potable y comunicación, posee la flamante instalación de gas natural en la puerta de su casa.

\section{REFERENCIAS}

BANKS, R.; R. BLUNDELL y A. LEWBEL (1997). "Quadratic Engel curves and consumer demand", The Review of Economics and Statistics 79 (4), pp. 527-539.

BLOW, L. (2003). "Explaining trends in UK households spending", Institute for Fiscal Studies, WP03/06.

BLUNDELL, R. W. y J. ROBIN (1999). "Estimation in large and disaggregated demand systems: and estimator for conditionally linear systems", Journal of Applied Econometrics 14, pp. 209-232.

DEATON, A.S. y J. MUELLBAUER (1980). "An almost ideal demand system", American Economic Review 70 (3), pp. 312-326.

DECOSTER, A. y F. VERMEULEN (1998). "Evaluation of the empirical performance of two-stage budgeting AIDS, QUAIDS and Rotterdam models based on weak separability", Center of Economic Studies, Discussion Paper Series DPS 98.08, Departement Economie - Katholieke Universiteit Leuven.

GARCIA, D. (2003). "El sistema casi ideal de demanda: un estado del arte", Ecos de Economía 16, pp. 77-94.

GOMEZ, A. (1996). "The welfare consequences of tariff rebalancing in the domestic gas market", Fiscal Studies 16, pp. 81-105. 
LABANDEIRA, X.; J. LABEAGA y M. RODRIGUEZ (2004). "Microsimulating the effects of household energy price changes in Spain", Mimeo, Fundación de Estudios de Economía Aplicada, http:// econwpa.wustl.edu:80/eps/pe/papers/0412/0412001.pdf.

LABANDEIRA, X., LABEAGA, J. y RODRIGUEZ, M. (2005). "A residencial energy demand system for Spain”, WP 2005-001, Mimeo, Fundación de Estudios de Economía Aplicada, http://webs.uvigo. es/xavier/papers/dtfedea.pdf.

LABANDEIRA, X. y A. LOPEZ (2002). "La imposición de carburantes de automoción en España: Algunas observaciones teóricas y empíricas", Hacienda Pública Española, Revista de Economía Pública, pp. 177-210.

LEE, E. y R. FORTHOFER (2006). Analyzing Complex Survey Data. Second Edition, Sage University Papers series on Quantitative Applications in the Social Sciences, $N^{\circ}$ 07-71. Thousand Oaks, CA: Sage.

MEDINACELI, M. (2005). "Consumo de Gas Natural en el Mercado Interno Urbano: ¿Beneficia a los Pobres? - Un análisis de microsimulación", Revista de Estudios Económicos y Sociales 4, "Estadísticas y Análisis", Instituto Nacional de Estadística, septiembre de 2005.

WEST, S. y R. WILLIAMS III (2002). "Estimates from a consumer demand system: Implications for the incidence of environmental taxes", National Bureau of Economic Research, Working Paper 9152, Cambridge - Massachusetts. 


\section{ANEXO 1}

\section{CALCULO DE LA FUNCION QAIDS}

Reemplazando:

$$
\begin{gathered}
\ln a\left(p_{t}\right)=\alpha_{0}+\sum_{i=1}^{n} \alpha_{i} \cdot \ln p_{i, t}+\frac{1}{2} \cdot \sum_{i=1}^{n} \sum_{j=1}^{n} \gamma_{i, j} \cdot \ln p_{i, t} \cdot \ln p_{j, t} \\
b\left(p_{t}\right)=\prod_{i=1}^{n} p_{i, t}^{\beta_{i}} \\
g\left(p_{t}\right)=\sum_{i=1}^{n} \lambda_{i} \cdot \ln p_{i, t}
\end{gathered}
$$

en

$$
\ln c(u, p)=\ln a\left(p_{t}\right)+\frac{u \cdot \ln b\left(p_{t}\right)}{1-u \cdot g\left(p_{t}\right)}
$$

Se obtiene:

$$
\ln c(u, p)=\alpha_{0}+\sum_{i=1}^{n} \alpha_{i} \cdot \ln p_{i, t}+\frac{1}{2} \cdot \sum_{i=1}^{n} \sum_{j=1}^{n} \gamma_{i, j} \cdot \ln p_{i, t} \cdot \ln p_{j, t}+\frac{u \cdot \ln \prod_{i=1}^{n} p_{i, t}^{\beta_{i}}}{1-u \cdot \sum_{i=1}^{n} \lambda_{i} \cdot \ln p_{i, t}}
$$

Que corresponde a la siguiente función indirecta de utilidad ${ }^{24}$ :

$$
\Psi=\left[\left(\frac{\ln m_{t}-\alpha_{0}-\sum_{i=1}^{n} \alpha_{i} \cdot \ln p_{i, t}-\frac{1}{2} \cdot \sum_{i=1}^{n} \sum_{j=1}^{n} \gamma_{i, j} \cdot \ln p_{i, t} \cdot \ln p_{j, t}}{\prod_{i=1}^{n} p_{i, t}^{\beta_{i}}}\right)^{-1}+\sum_{i=1}^{n} \lambda_{i} \cdot \ln p_{i, t}\right]^{-1}
$$

Utilizando el lema de Shepard se obtiene:

$$
w_{i, t}=\alpha_{i}+\sum_{j=1}^{n} \gamma_{i, j} \cdot \ln p_{j, t}+\beta_{i} \cdot \log \left[\frac{m_{t}}{a\left(p_{t}\right)}\right]+\frac{\lambda_{i}}{b\left(p_{t}\right)} \cdot \log \left[\frac{m_{t}}{a\left(p_{t}\right)}\right]^{2}
$$

24 Decoster y Vermeulen (1998). 


\section{ANEXO 2}

\section{RESULTADOS DE LA ESTIMACION}

\begin{tabular}{|c|c|c|}
\hline Variable & Gas Natural & GLP \\
\hline \multirow[t]{2}{*}{ Constante } & $-0,00000222$ & 0,25795650 \\
\hline & 0,00058421 & 0,00208432 \\
\hline \multirow[t]{2}{*}{ Error variable instrumental } & $\mathbf{0 , 0 0 0 0 0 0 0 1}$ & 0,00000003 \\
\hline & 0,00000000 & 0,00000001 \\
\hline \multirow[t]{2}{*}{$\lambda$} & 0,00437475 & $-0,01401022$ \\
\hline & 0,00019765 & 0,00070963 \\
\hline \multirow[t]{2}{*}{ Vivienda propia $(1=$ Sí, $0=$ No $)$} & 0,00019399 & 0,00064347 \\
\hline & 0,00001153 & 0,00004141 \\
\hline \multirow[t]{2}{*}{ Número miembros familia } & $-0,00000814$ & 0,00070512 \\
\hline & 0,00000299 & 0,00001074 \\
\hline \multirow[t]{2}{*}{$\%$ de familias con instalación de gas natural } & 0,00248433 & 0,00423810 \\
\hline & 0,00016504 & 0,00059267 \\
\hline \multirow[t]{2}{*}{ Número de cocinas en el hogar } & $-0,00019943$ & 0,00347891 \\
\hline & 0,00001561 & 0,00005604 \\
\hline \multirow[t]{2}{*}{ Número de hornos en el hogar } & 0,00004487 & 0,00045511 \\
\hline & 0,00001578 & 0,00005667 \\
\hline \multirow{2}{*}{ Número de bienes que utilizan energía eléctrica } & 0,00005175 & 0,00004257 \\
\hline & 0,00000287 & 0,00001029 \\
\hline \multirow{2}{*}{ Variable ficticia Distribuidoras Privadas } & 0,00004977 & $-0,00115666$ \\
\hline & 0,00005645 & 0,00020245 \\
\hline \multirow[t]{2}{*}{ Log precio gas natural } & $-0,00000029$ & $-0,00097619$ \\
\hline & 0,00010500 & 0,00037711 \\
\hline \multirow[t]{2}{*}{ Log precio GLP } & 0,00057864 & $-0,00486054$ \\
\hline & 0,00014577 & 0,00051871 \\
\hline \multirow[t]{2}{*}{ Log (gasto total) } & 0,00014345 & $-0,04730025$ \\
\hline & 0,00012642 & 0,00044273 \\
\hline \multirow[t]{2}{*}{$\log (\text { gasto total })^{2}$} & $-0,00002149$ & $\mathbf{0 , 0 0 2 1 0 2 3 3}$ \\
\hline & 0,00000772 & 0,00002628 \\
\hline $\mathrm{R} 2$ & 0,073311 & 0,447430 \\
\hline R2 ajustado & 0,073266 & 0,447400 \\
\hline $\mathrm{F}$ & $1.623,8$ & $1.623,8$ \\
\hline
\end{tabular}


RESULTADOS DE LA ESTIMACION PROBIT DE LA VARIABLE $\lambda$ :

\begin{tabular}{|ccccc|}
\hline \multicolumn{5}{c|}{ Variable Dependiente: GN } \\
\hline Variable & Coeficiente & Error Estándar & Estadístico z & Prob. \\
\hline C & $-2,182276$ & 0,048775 & $-44,74195$ & 0,0000 \\
COBERTURA & 2,680364 & 0,142886 & 18,75878 & 0,0000 \\
COCINA & 0,148615 & 0,033527 & 4,432739 & 0,0000 \\
HORNO & 0,168801 & 0,034700 & 4,864578 & 0,0000 \\
INGRESO & $3,33 \mathrm{E}-05$ & $2,72 \mathrm{E}-06$ & 12,24616 & 0,0000 \\
MIEM & $-0,025538$ & 0,008123 & $-3,144122$ & 0,0017 \\
TJA & 0,408808 & 0,054376 & 7,518145 & 0,0000 \\
CBBA & 0,028452 & 0,044275 & 0,642610 & 0,5205 \\
\hline Obs. con Dep $=0$ & 22.122 & Total observaciones & 23.103 \\
Obs. con Dep $=1$ & 981 & & \\
\hline
\end{tabular}

Donde:

COBERTURA $=$ Porcentaje de familias en el departamento con acceso a gas natural.

COCINA $=$ Número de cocinas que posee la familia.

HORNO = Número de hornos que posee la familia.

INGRESO = Ingreso total de la familia.

MIEM $\quad$ Número de miembros en la familia.

TJA $\quad=$ Variable ficticia, toma el valor de 1 cuando la familia pertenece al departamento de Tarija.

CBBA = Variable ficticia, toma el valor de 1 cuando la familia pertenece al departamento de Cochabamba.

\section{ANEXO 3 \\ ESPECIFICACION ALTERNATIVA}

Las especificaciones utilizadas son:

$$
\begin{aligned}
& g n=\alpha_{0}^{*}+\sum_{j=1}^{k} \alpha_{j}^{*} x_{j}+e p_{g n}^{*} \cdot \ln p_{g n}+e p_{g l p}^{*} \cdot \ln p_{g l p}+e i_{g n} \ln m \\
& g l p=\alpha_{0}^{* *}+\sum_{j=1}^{k} \alpha_{j}^{* *} x_{j}+e p_{g n}^{* *} \cdot \ln p_{g n}+e p_{g l p}^{* *} \cdot \ln p_{g l p}+e i_{g l p} \ln m
\end{aligned}
$$

Donde:

$g n=$ consumo de gas natural expresado en miles de pies cúbicos.

$g l p=$ consumo de GLP expresado en kilogramos.

$x_{j}=$ características de los hogares.

$p_{g n}=$ precio del gas natural

$p_{g l p}=$ precio del GLP

$m$ = gasto total de la familia. 
La estimación fue realizada dividiendo la muestra en dos grupos, en el primero sólo se consideran las familias que reportan consumo positivo de gas natural; en el segundo, aquellas que lo hacen para el GLP. También se incorporó, dentro el vector de variables $x_{j}$ la instrumentación de la variable gasto total con el ingreso total del hogar. Los resultados se presentan a continuación:

Variable Dependiente: LOG(DGN)

Muestra: 1981

\begin{tabular}{|crrrr|}
\hline Variable & Coeficiente & Error Estándar & t-Estadístico & Prob. \\
\hline C & $-0,873180$ & 1,406490 & $-0,620822$ & 0,5349 \\
VIV & 0,068249 & 0,029447 & 2,317710 & 0,0207 \\
MIEM & 0,039760 & 0,007560 & 5,259037 & 0,0000 \\
PORFAM & 0,155478 & 0,222665 & 0,698262 & 0,4852 \\
COCINA & 0,136780 & 0,044638 & 3,064173 & 0,0022 \\
HORNO & 0,093450 & 0,030994 & 3,015099 & 0,0026 \\
PRIVADA & 0,094447 & 0,314373 & 0,300430 & 0,7639 \\
VI & $1,73 \mathrm{E}-05$ & $6,61 \mathrm{E}-06$ & 2,624332 & 0,0088 \\
LOG(P1R) & $-1,005856$ & 0,603680 & $-1,666206$ & 0,0960 \\
LOG(P2R) & 3,621452 & 0,825314 & 4,387967 & 0,0000 \\
LOG(GT) & 0,187922 & 0,027618 & 6,804349 & 0,0000 \\
\hline Estadísticos ponderados & & & & \\
\hline R-cuadrado & 0,441306 & & \\
Durbin-Watson & 1,150654 & & & \\
\hline Estadísticos no ponderados & & & \\
\hline R-cuadrado & 0,134947 & & \\
Durbin-Watson & 0,391372 & & \\
\hline
\end{tabular}


Variable Dependiente: LOG(DGLP)

Muestra: 119990

\begin{tabular}{|crlll|}
\hline Variable & Coeficiente & Error Estándar & t-Estadístico & Prob. \\
\hline C & $-0,478289$ & 0,210803 & $-2,268896$ & 0,0233 \\
VIV & 0,078321 & 0,004284 & 18,28309 & 0,0000 \\
MIEM & 0,058756 & 0,001215 & 48,37573 & 0,0000 \\
PORFAM & 0,309636 & 0,041175 & 7,520054 & 0,0000 \\
COCINA & $-0,003068$ & 0,006877 & $-0,446134$ & 0,6555 \\
HORNO & $-0,026485$ & 0,006127 & $-4,322436$ & 0,0000 \\
ELEC & 0,004864 & 0,001096 & 4,438404 & 0,0000 \\
PRIVADA & $-0,400821$ & 0,048717 & $-8,227527$ & 0,0000 \\
VI & $4,09 \mathrm{E}-07$ & $1,02 \mathrm{E}-06$ & 0,402114 & 0,6876 \\
LOG(P1R) & 0,810229 & 0,092885 & 8,722968 & 0,0000 \\
LOG(P2R) & $-0,492304$ & 0,125917 & $-3,909763$ & 0,0001 \\
LOG(GT) & 0,079047 & 0,005400 & 14,63844 & 0,0000 \\
\hline Estadísticos ponderados & & & & \\
\hline R-cuadrado & 0,983016 & & & \\
Durbin-Watson & 0,697111 & & & \\
\hline Estadísticos no ponderados & & & \\
\hline R-cuadrado & 0,191732 & & \\
Durbin-Watson & 0,233714 & & \\
\hline
\end{tabular}

Donde:

VIV $=$ Variable ficticia que toma el valor de 1 cuando la familia es dueña de la vivienda.

MIEM = Número de miembros en la familia.

PORFAM $=\lambda$.

COCINA $=$ Número de cocinas en la familia.

HORNO = Número de hornos en la familia.

PRIVADA = Variable ficticia que toma el valor de 1 cuando la familia vive en un departamento en el cual la administración de la red de distribución de gas natural la realiza el sector privado.

VI $=$ Variable instrumental del ingreso total.

$\operatorname{LOG}(\mathrm{GT})=$ Logaritmo del gas total.

$\mathrm{LOG}(\mathrm{P} 1 \mathrm{R})=$ Logaritmo del precio relativo del gas natural.

$\mathrm{LOG}(\mathrm{P} 2 \mathrm{R})=$ Logaritmo del precio relativo del GLP.

ELEC $=$ Número de bienes que la familia posee y funcionan con energía eléctrica. 